Plant Tissue Cult. \& Biotech. 23(2): 133-146, 2013 (December)

$\underline{\text { PTC\&B }}$

\title{
In vitro Root Culture : An Alternative Source of Bioactives in the Rare Aphrodisiac Herb Chlorophytum borivilianum Sant et Fern
}

\author{
Sautrik Basu and Timir Baran Jha* \\ Department of Botany, Maulana Azad College, Kolkata-13, India \\ Key words: C. borivilianum, Root culture, Bioactives, Rare aphrodisiac
}

\begin{abstract}
Chlorophytum borivilianum is a rare, near-endemic high value root crop. The fasciculated roots of the plant have the potential to synthesize valuable bioactives having aphrodisiac, anti stress and immuno modulatory properties. The present investigation describes an alternative protocol for the development of a stable, fast growing, non-transformed root culture system in C. borivilianum and the simultaneous quantitative analysis of total steroidal saponins, amino acids and sugars. Liquid MS fortified with various concentrations and combinations of BA $(0.44-8.8 \mu \mathrm{M}), \mathrm{Kn}(0.23 \mu \mathrm{M})$ and NAA $(0.27-5.4 \mu \mathrm{M})$ was effectively used for the development of root culture system. High performance thin layer chromatography (HPTLC) was used for quantification and comparative analysis of bioactives from in vitro and in vivo grown tuberous roots.
\end{abstract}

\section{Introduction}

Chlorophytum borivilianum Sant et Fern (Liliaceae) known commonly as "safed musli" is a rare aphrodisiac herb valued for its dried fasciculated storage roots. The plant has been used extensively for centuries as an important ingredient of herbal tonics prescribed in the Ayurvedic and Unani systems of medicine (Oudhia 1999). The storage roots of C. borivilianum are the major sources of steroidal and triterpenoidal saponins, sugars, polysaccharides and amino acids. The roots are also a rich source of over 25 alkaloids, proteins, vitamins, steroids, phenolics, gallotannins, mucilage and resins (Thakur et al. 2008). The plant supports an annual growth habit and has a very short life cycle (90 - 100 days) (Maiti and Geetha 2005). Ever growing market demand coupled with rapid

*Author for correspondence. P.G. Department of Botany, Barasat Govt. College, Barasat, Kolkata-700124, India. <tbjha2000@yahoo.co.in>. 
habitat loss, ruinous harvesting practices, prolong seed dormancy (nearly ten months) and poor germination rate (14 - 16\% only) have made C. borivilianum a rare plant (Maiti and Geetha 2005). Thus development of faster propagation techniques and formulation of alternative strategies for the production of bioactive molecules through biotechnological interventions are essential for improvement and conservation of the species. There are a few reports describing tissue culture based propagation of C. borivilianum (Purohit et al. 1994, Suri et al. 1999, Dave et al. 2003, Basu and Jha 2007, Rizvi et al. 2007). But reports on specific characterization and quantification of root specific bioactive compounds from in vitro culture systems are meager or none. Since Chlorophytum roots are the principle material for many herbal formulations and can be consumed directly as a raw drug, the development of a fast growing root culture system can offer us a unique opportunity of manufacturing root drugs in the laboratory and can substantially reduce the dependence on field cultivation. Root cultures since the early days of tissue culture research are considered as reliable, alternative sources for the production of valuable bioactives. Reports describing in vitro culture of excised roots are available mainly in case of dicotyledonous plants, but root culture in monocots is difficult particularly in those which form bulb, corm or tuber (Ghosh et al. 2002). Consequently published reports are very few in case of monocots (Ghosh et al. 2002, 2006, 2007). Considering all the above mentioned facts the primary objective of the present report was to establish a stable, fast growing, non-transformed root culture system in C. borivilianum and to present a comprehensive data on quantitative analysis of some important bioactive compounds from in vitro and in vivo grown roots employing HPTLC.

\section{Materials and Methods}

The hypocotyl segments of the in vitro germinated seedlings belonging to two different populations [Narendrapur (W.B.) designated as CB-1 and Mt. Abu (Rajasthan) designated as CB-2] were used as primary explants. Seeds were germinated in vitro following previously published methods (Basu and Jha 2007).

Full strength liquid MS supplemented with 3\% (w/v) sucrose was used for initiation of root culture. The media were fortified with different concentrations and combinations of BA $(0.44-8.8 \mu \mathrm{M}), \mathrm{Kn}(0.23 \mu \mathrm{M})$ and NAA $(0.27-5.4 \mu \mathrm{M})$. $30 \mathrm{ml}$ of liquid media was dispensed in $250 \mathrm{ml}$ conical flasks. Cultures were maintained at $22 \pm 1^{\circ} \mathrm{C}$ and a relative humidity of $50-60 \%$ under cool white fluorescent tubes (Philips, India) emitting $32 \times 10^{8} \mathrm{M} \mathrm{m}^{2} / \mathrm{s}$ for 16/8 hrs light/dark periods. The liquid cultures were maintained under continuous agitation at 80 rpm on a gyratory shaker (Eyela, China). The effect of different growth regulators on root/tuber formation was recorded at regular intervals. 
All experiments were repeated thrice with multiple replicates. Statistical difference between mean tabulated values were estimated $(p \leq 0.05)$ using DMRT with Statistica software Version 10.0.

For chemical analysis about 6 gm of dry and powdered root tissue were taken from each donor population as well as from the in vitro grown roots. Authentic spirostane saponin, sapogenins, arginine and fructose were obtained from Natreon-Inc Kolkata (India), Dept of Botany, University of Calcutta and Sigma Aldrich (USA). All solvents (HPLC grade) and reagents (GR grade) were obtained from E. Merck (India).

Extraction of saponins and sapogenins were carried out following previously published methods (Thakur et al. 2007) with minor modifications. For extraction of crude saponins defatted root samples were individually extracted under reflux with $80 \% \mathrm{MeOH}$ (three times). The $\mathrm{MeOH}$ extracts were pooled and concentrated under reduced pressure to obtain a dark colored residue. The residue of each sample was dissolved in water and then partitioned with $n$-butanol. The $n$-butanol layer was separated, concentrated under reduced pressure and saponins were precipitated with diethyl ether. The precipitated saponins were eventually collected after centrifugation and dissolved in water. Chromatography and quantification of saponins were carried out from these samples. The crude saponins were hydrolysed with $2 \mathrm{~N} \mathrm{HCl}$ to release the aglycones (sapogenins) and also the glycone fractions. Chromatography and quantification of individual saponin aglycones and sugars were performed following the methods of Jin et al. (2004) and Xin et al. (2003), respectively with minor modifications. Extraction, chromatography and quantification of amino acids was performed following the method of Hess and Sherma (2004).

The HPTLC system consisted of a CAMAG (Muttenz, Switzerland) Linomat5 automatic sample applicator (with nitrogen flow) and CAMAG TLC scanner-3 equipped with WINCATS software (version: 1.4.4.6337). The stationary phase consisted of pre-coated silica gel $60 \mathrm{~F}_{254}$ plates $(20 \mathrm{~cm} \times 10 \mathrm{~cm}$; with $0.25 \mathrm{~mm}$ layer thickness: Merck KGaA; 1.05554. 0007). Samples were applied to the plates as bands $6 \mathrm{~mm}$ wide, with $10 \mathrm{~mm}$ distance between tracks, by means of Linomat- 5 automatic sample applicator equipped with a $100 \mu$ l Hamilton syringe. The mobile phase used for saponins consisted of a mixture of chloroform: methanol: acetic acid: water $(60: 30: 10: 8)$ and for sapogenins two different mobile phases were used [Benzene: isopropanol (70:30) was used for sarsasapogenin and benzene: methanol $(70: 30)$ was used for tigogenin and ruscogenin]. The mobile phase for amino acids consisted of a mixture of butanol: acetic acid: water $(4: 1$ : 2) and for sugars a gradient system was followed as proposed by Xin et al. (2003). For saponins the plates were scanned initially at $210 \mathrm{~nm}$ and then at 530 
nm after derivatization with vanillin-sulphuric acid reagent. For sapogenins the TLC plates were scanned at $490 \mathrm{~nm}$ after derivatization with vanillin-sulphuric acid reagent. For amino acids scanning was done at $490 \mathrm{~nm}$ after derivatization with $0.3 \%$ ninhydrin solution and for sugars the plates were scanned at $530 \mathrm{~nm}$ after derivatization with a reagent solution consisting of $0.5 \mathrm{~g}$ of 4-amino benzoic acid, $9 \mathrm{ml}$ of glacial acetic acid. Ten $\mathrm{ml}$ of water and $0.5 \mathrm{ml}$ of $85 \% \mathrm{H}_{3} \mathrm{PO}_{4}$ (Xin et al. 2003). The densitograms and spectra obtained were matched with that of the external standards used and peak areas were evaluated by the help of CAMAG TLC Scanner 3 equipped with WINCATS software. Total saponins were quantified on the basis of the standard curve obtained from the spirostane marker $\left[Y=1386+2.82 X\left(r^{2}=0.921\right)\right]$ while the sapogenins were quantified on the basis of their individual calibration curves [Sarsasapogenin: $\mathrm{Y}=45.94+0.820 \mathrm{X}$ $\left(\mathrm{r}^{2}=0.998\right)$; tigogenin: $\mathrm{Y}=291.4+603.1 \mathrm{X}\left(\mathrm{r}^{2}=0.96\right)$; Ruscogenin: $\mathrm{Y}=244.7+4.56 \mathrm{X}$ $\left.\left(r^{2}=0.986\right)\right]$. Total amino acids were quantified on the basis of the calibration curve of L-arginine $\left[\mathrm{Y}=1315+2.538 \mathrm{X}\left(\mathrm{r}^{2}=0.991\right)\right]$ and total sugars were quantified on the basis of the calibration curve of D-fructose $\left[Y=6671+9.875\left(\mathrm{r}^{2}=\right.\right.$ 0.976)]. All experiments were repeated thrice and validated for linearity, precession (intra- and inter day), specificity, limit of detection (LOD) and limit of quantification (LOQ) following ICH guidelines.

\section{Results and Discussion}

Successful establishment of contamination free cultures did not pose any major problems since hypocotyl segments of in vitro germinated seeds containing a root primordia were used as explants (Fig. 1a). Successful establishment of a stable non-transformed root culture system was also effectively achieved on liquid MS fortified with various growth regulators (Table 1). Rapid root growth and differentiation started after 15 days of culture (Fig. 1b) and maximum growth of roots was noticed in between 6th and 12th weeks (Fig. 1c, d).

Liquid MS supplemented with $8.80 \mu \mathrm{M}$ BA, $0.23 \mu \mathrm{M}$ Kn and 5.40 $\mu \mathrm{M}$ NAA was highly conducive for in vitro root formation and yielded 25.7 and 29.7 roots from a single explant of the West Bengal and Rajasthan population, respectively after 12 weeks of culture (Table 1) under 16/8 hrs light/dark incubation. Interestingly, light incubation did not inhibit root growth and differentiation as documented in many other systems (Norton and Boe 1982). However, the roots obtained on MS liquid medium under the regime of different growth regulators (BA, Kn and NAA) exhibited a very different growth pattern, colour and peculiar lateral branching (Fig. 1d and g) which is highly unusual for monocots and has not been reported earlier. Like all other monocotyledonous plant Chlorophytum 

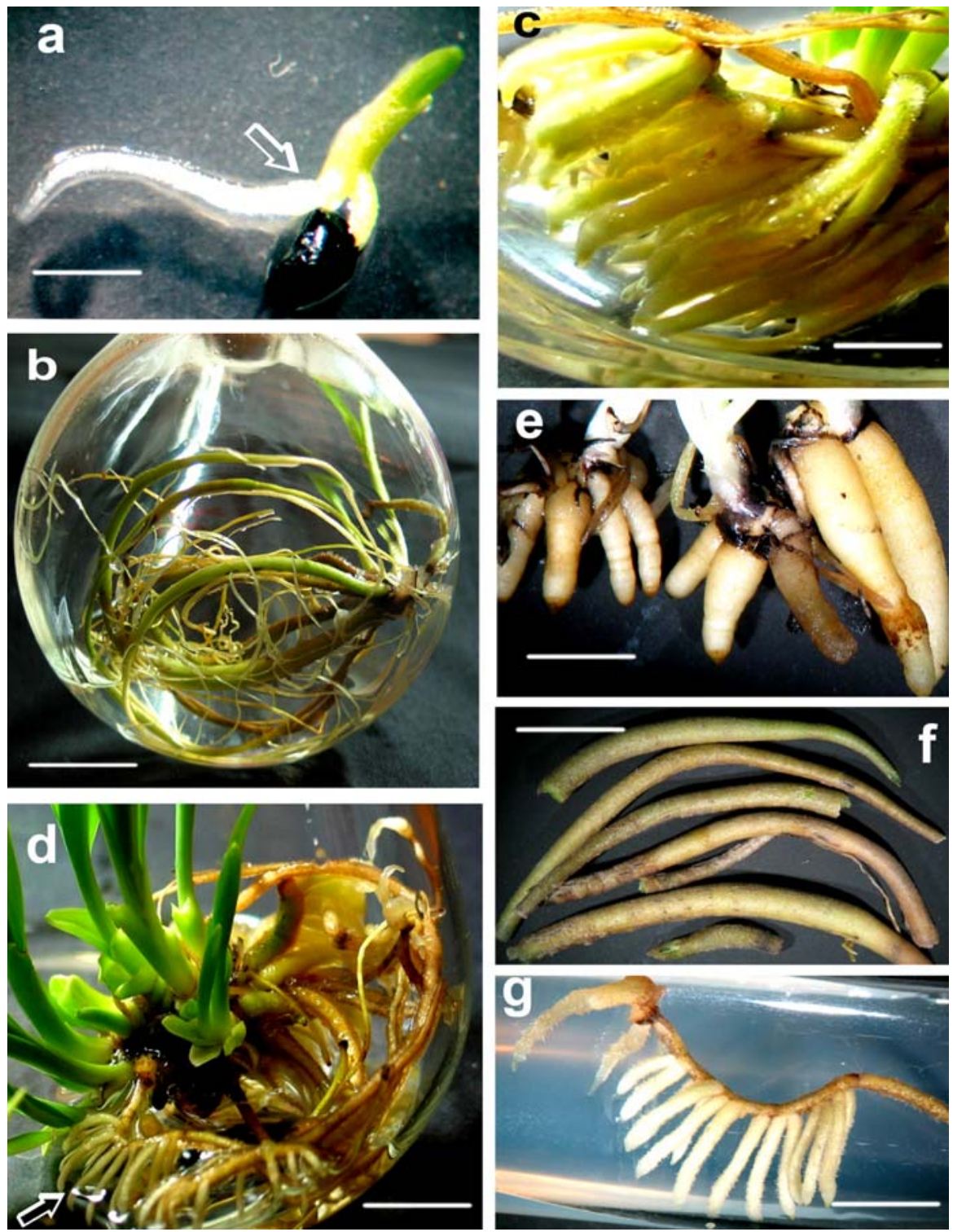

Fig.1. (a) Hypocotyl segment (indicated by arrow) of a single in vitro germinated seeds used as primary explant. (b) Root growth in the liquid medium observed after 15 days of inoculation (in CB-1). (c) Profuse root growth obtained in the liquid medium after six weeks of culture in the Rajasthan population (CB-2). (d) Formation of characteristic laterals after 12 weeks of culture in the West Bengal population (CB-1) which in course of time appeared like miniature tubers. (e) Formation of stout, light coloured divergent type of roots with blunt ends and better storage quality in the Rajasthan population. (f) Formation of long dark brown to greenish, cylindrical, convergent type of fleshy roots in the West Bengal population (CB-1) after 12 weeks of culture. (g) A portion of the cultured root showing characteristic branching in the West Bengal population (CB-1). Bars $=0.5 \mathrm{~cm}$. 


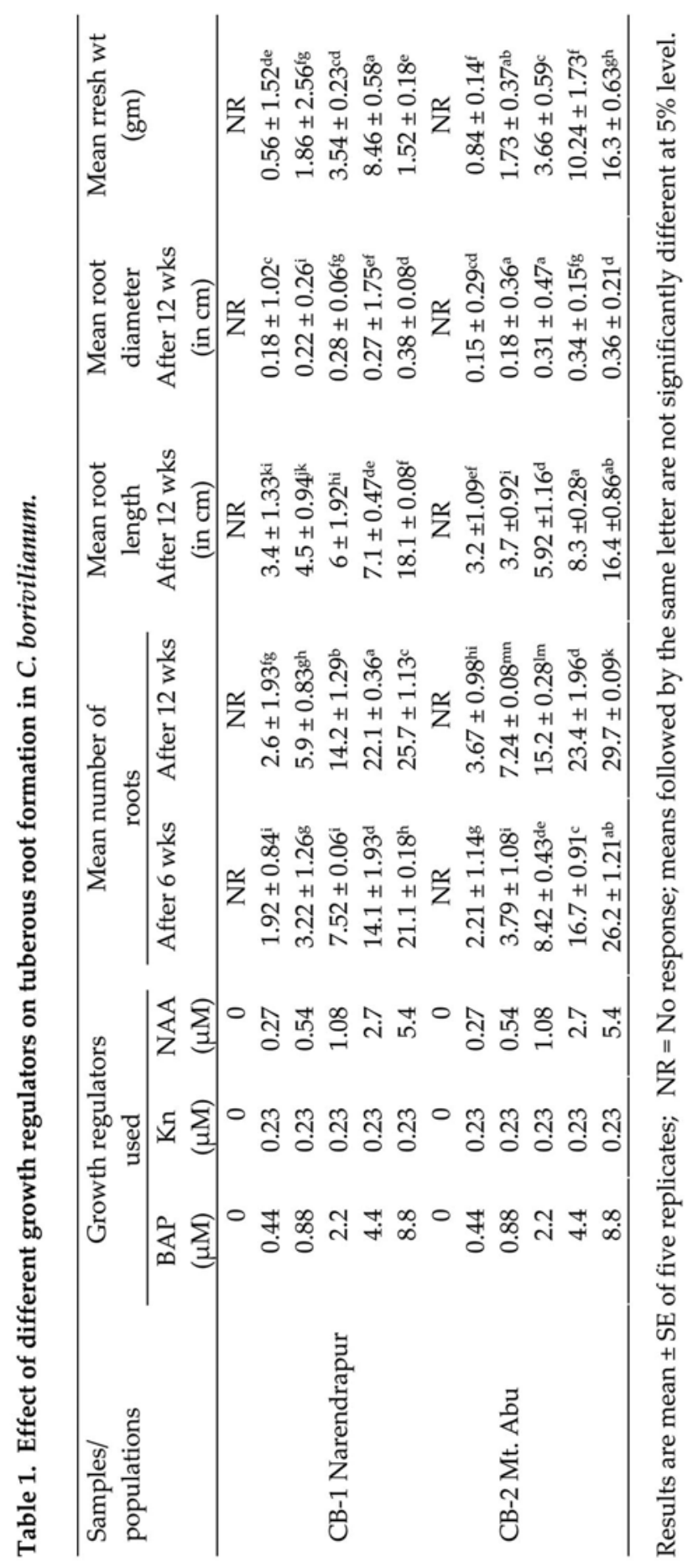


also possesses fibrous roots which in course of time are modified into sessile fasciculated tubers comprising the economically useful part. Studies conducted earlier has revealed the existence of high variability in terms of colour, length and shape of fleshy roots of C. borivilianum (Maiti and Geetha 2009). In the present investigation we observed the formation of dark brown to greenish, cylindrical, convergent type of fleshy roots with tapering ends in CB-1 (Fig. 1b and f) and stout, light coloured divergent type of roots with blunt ends and better storage quality in case of CB-2 (Fig. 1e). Although the roots of the Rajasthan population (CB-2) when grown in vitro exhibited similar morphology with that of the donor, the in vitro grown roots of the West Bengal population exhibited the formation of peculiar laterals which in course of time appeared like miniature tubers (Fig. 1d and g). This unusual growth pattern may be attributed to prolonged growth in liquid medium under slow agitation. It has been reported that continuous agitation obstructs apical dominance which ultimately leads to increased adventitious branching (Wawrosch et al. 2005, Kongbangkerd and Wawrosch 2003). In addition to the steady production of root biomass (Table 1 ), production of shoots was also noticed in long term cultures (12 weeks or more, Fig. 1d) in the same medium supplemented with BA, Kn and NAA thus making the protocol a single step one for the production of root biomass as well as microshoots. No decline in root biomass yield was noted even after 12 weeks of culture (beyond three subculture passages) and the in vitro grown roots produced lateral branches continuously. The role of auxins in induction and development of roots is well elucidated but relatively little is known about the role of cytokinin(s) on lateral root development. Even reports describing the inhibitory effect of cytokinin(s) on lateral root formation are available (Bottger 1978, Goodwin and Morris 1979, Wightman et al. 1980). In the present study, however, no such inhibitory effect of cytokinins was noticed and production of healthy fasciculated roots took place under the combined influence of BA and $\mathrm{Kn}$. Reports published earlier have demonstrated that $\mathrm{Kn}$ promotes the development of roots (Hill and Lawrence 1985) and a low dose of $\mathrm{Kn}$ in combination with NAA has been found to be highly conducive for induction of roots from young leaf explants of Ocimum sanctum (Shilpa et al. 2010). Our reports are also in agreement with the previous reports describing the promotive effects of BA and $\mathrm{Kn}$ on root growth and proliferation. It is interesting to mention here that the in vitro grown roots (of both populations) when excised and subcultured on semi solid/liquid medium devoid of growth regulators lost their morphogenic potential and became necrotic within three weeks of inoculation.

Storage roots of "safed musli" has been extensively used for centuries as an important ingredient of many herbal tonics and are often consumed as a raw 
drug by rural and tribal communities. In spite of its immense popularity, ever increasing global demand and high market value phytochemical analysis in $C$. borivilianum has been mainly restricted to isolation, purification and characterization of saponins and polysachharides (Acharya et al. 2008, 2009, Narasimhan et al. 2006). No attempts have been made so far, for the development

Table 2. Major root specific bioactives detected in root cultures of C. borivilianum and their donor populations.

\begin{tabular}{lcccc}
\hline \multirow{2}{*}{$\begin{array}{l}\text { Major root specific } \\
\text { bioactives }\end{array}$} & \multicolumn{4}{c}{$\begin{array}{c}\text { Roots of different donor populations and their in vitro } \\
\text { counterparts analysed }\end{array}$} \\
\cline { 2 - 5 } & CB-1 & CB-2 & CBRC-1 & CBRC-2 \\
\hline $\begin{array}{l}\text { Steroidal saponins } \\
\text { (\% per gm of dry wt.) }\end{array}$ & $26.73 \pm 0.04$ & $37.36 \pm 0.08$ & $18.9 \pm 1.18$ & $20.0 \pm 0.07$ \\
Total sugars & $16.83 \pm 1.89$ & $18.16 \pm 1.97$ & $19.72 \pm 1.76$ & $24.66 \pm 0.48$ \\
(\% per gm of dry wt.) & & & & \\
Total amino acids & $3.53 \pm 0.13$ & $4.89 \pm 1.36$ & $17.48 \pm 0.07$ & $21.09 \pm 1.79$ \\
(\% per gm of dry wt.) & & & & \\
Ruscogenin (mg/g) & $0.37 \pm 0.03$ & $0.40 \pm 0.002$ & $0.069 \pm 0.071$ & $0.084 \pm 0.034$ \\
$\begin{array}{l}\text { Tigogenin (mg/g) } \\
\text { Sarsasapogenin (mg/g) }\end{array}$ & $0.88 \pm 0.02$ & $1.26 \pm 0.05$ & $0.308 \pm 0.21$ & $0.69 \pm 0.032$ \\
\hline
\end{tabular}

Results are mean \pm SE of three replicates. CBRC: Cultured roots of $C$. borivilianum.

of transformed/non transformed root cultures, which can serve as an alternative for the production of important root specific bioactives irrespective of season. Taking into account the inadequacies left in the previous studies we have attempted to develop an in vitro protocol for steady supply of tuberous roots and also to present a comprehensive quantitative analysis of the field grown roots and their in vitro counterparts in terms of saponins, sapogenins, aminoacids and sugars. Saponins are secondary metabolites of glycosidic nature possessing a wide range of pharmacological activities including expectorant, antiinflammatory, cytotoxic, haemolytic, vasoprotective, hypocholesterolemic, immunomodulatory, hypoglycaemic, molluscicidal, antifungal, antiparasitic and many others (Sparg et al. 2004, Sahu et al. 2008). Amino acids on the other hand are commonly used nutritional supplements having several health restorative properties. Phytochemical analysis of in vitro grown roots of both the populations (CBRC-1 and 2) revealed the presence of substantial amount of saponins (18.9 and 20\% in CBRC-1 and 2) [Fig. 2 (ii) \& (iv)], amino acids (17.48 and $21.09 \%$ in CBRC-1 and 2) [Fig.2a (ii) \& (iv)], sugars (19.72 and $24.66 \%$ in CBRC-1 and 2) and three sapogenins [Sarsasapogenin (0.157 and $0.164 \mathrm{mg} / \mathrm{g}$ in CBRC-1 and 2), tigogenin (0.308 and $0.69 \mathrm{mg} / \mathrm{g}$ in CBRC-1 and 2) and ruscogenin (0.069 and $0.084 \mathrm{mg} / \mathrm{g}$ in CBRC-1 and 2). Comparative results are presented in 
Table 2. Reports describing the production and quantification of saponins from cultured cells and tissues are restricted mainly to the genus Panax (Furuya et al. 1983, Chi et al. 1989, Choi et al. 1994 and Wu et al. 1999) and Primula (Okrslar et al. 2007). A few reports on quantification of saponins from transformed roots of Panax ginseng are also available (Yoshikawa et al. 1987, Hwang et al. 1991) but reports describing quantification of saponins and sapogenins from in vivo/in vitro cultured roots of $C$. borivilianum are rare. Extensive perusal of literature reveals that although considerable work has been done on the saponins of other Chlorophytum species (Mimaki et al. 1996, Tandon and Shukla 1997, Qiu et al. 2000) there is a paucity of scientific work on specific characterization, quantification and standardization of saponins, sapogenins and other important bioactives of $C$. borivilianum. Presence of the anabolic amino acid L-arginine in cultured roots and their donor populations is a new finding which also has not been reported earlier. HPTLC profiles depicted in Fig. 2a (i-iv) clearly reveals the predominance of L-arginine in all the investigated samples. After quantification, it was realized that the in vitro grown roots are much superior in terms of Larginine. Tuberous roots of the donor populations (CB 1 and 2) were found to contain 2.66 and $3.46 \mathrm{mg} / \mathrm{g}$ L-arginine respectively while the amount of Larginine in the cultured roots (CBRC-1 and 2) were 3.83 and $4.89 \mathrm{mg} / \mathrm{g}$, respectively. This significant increase in the amino acid content may be attributed to the defined nutritional and hormonal regimes in the culture medium. Arginine is a bitter tasting anabolic amino acid (conditionally essential) found in plant/animal proteins whose main function is to increase nitric oxide (NO) production in human body. NO relaxes and dialates blood vessels, promoting better blood circulation to the heart, brain and bladder (Boradhust and Duke 1998). Aginine supplements are helpful in the treatment of angina, high cholesterol, hypertension, heart attack and stroke. It is also essential for the increased output of human growth hormone, which plays a vital role in fertility. Appreciable amount of sugars have also been detected in all the samples (Table 2) which is a typical characteristic of Chlorophytum roots. The percentage of total sugars has been found nearly identical in all the investigated samples (Table 2). The yields of saponins and sugars from elite accessions of C. borivilianum as reported previously by Bordia et al. 1995 are $2-17 \%$ and $40 \%$, respectively. The present study, however for the first time reports a much higher amount of saponins (37.36\% per gm of dry wt., in the population collected from Rajasthan) and lower amount of sugar (18.16\% per g dry wt.) in the roots of field grown donor plants. Present results are also indicative of the fact that in vitro cultured roots are not only capable of synthesizing appreciable amount of saponins $(20 \%$ per g dry wt.), but can also serve as an excellent source of free amino acids 

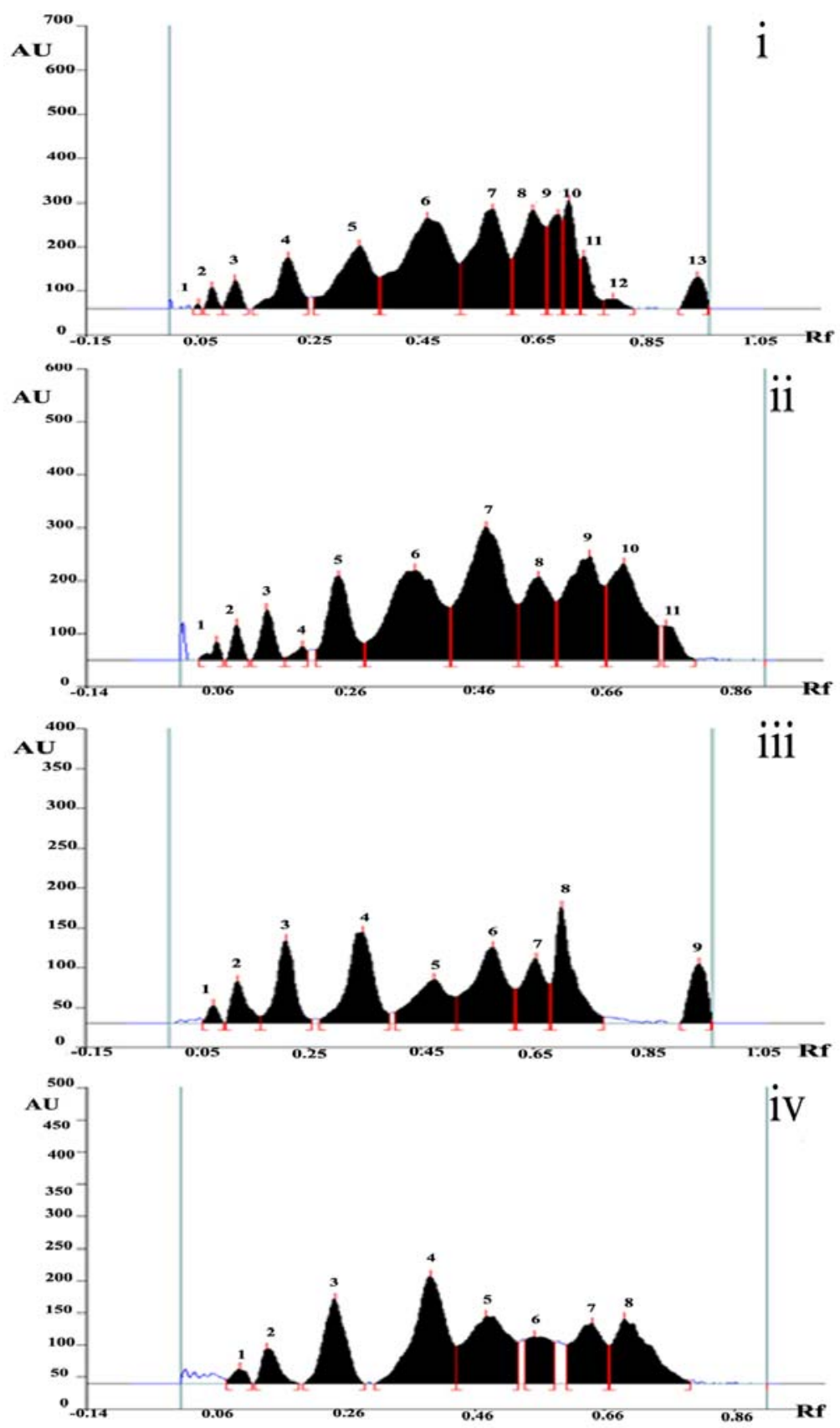

Fig. 2. (i) HPTLC chromatogram showing the saponin profile of the donor population collected from West Bengal (CB-1). (ii) Saponin profile obtained from the in vitro cultured roots of $C$. borivilianum (West Bengal population). (iii) HPTLC chromatogram showing the saponin profile of the donor population collected from Rajasthan (CB-2). (iv) Saponin profile obtained from the in vitro cultured roots of $C$. borivilianum (Rajasthan population). 

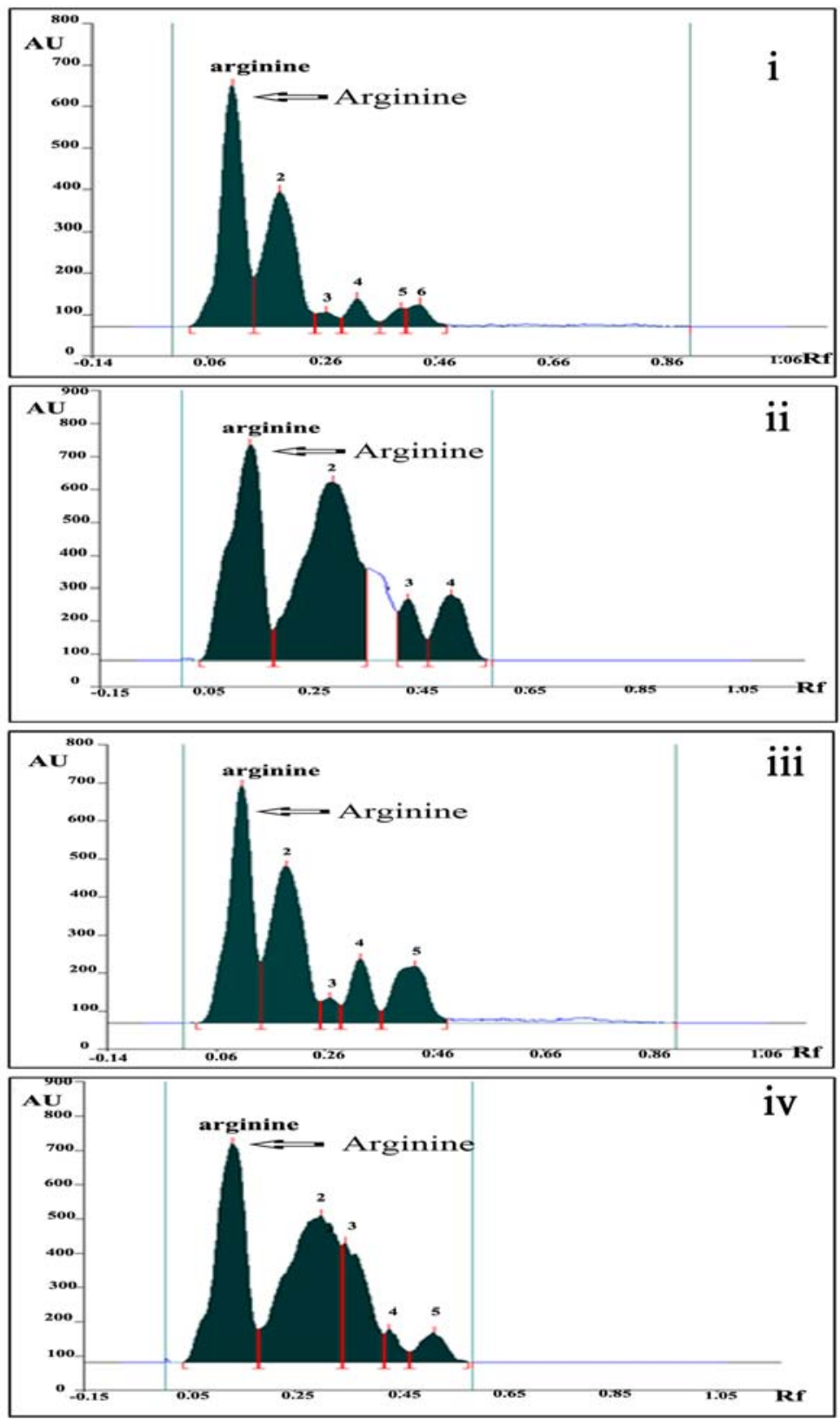

Fig.2a (i) HPTLC chromatogram showing the amino acid profile of the donor population collected from West Bengal (the peak for arginine) at $\mathrm{R}_{\mathrm{f}} 0.34$ has been indicated by arrow. (ii) HPTLC chromatogram showing the amino acid profile of the donor population collected from Rajasthan (the peak for arginine has been indicated by arrow). (iii) Amino acid profile obtained from the in vitro cultured roots of $C$. borivilianum (West Bengal population) [the peak for arginine has been indicated by arrow]. (iv) Amino acid profile obtained from the in vitro cultured roots of $C$. borivilianum (Rajasthan population) [the peak for arginine has been indicated by arrow]. 
(L-arginine in particular). Chemical fingerprinting using HPTLC in recent years has emerged as an important and effective tool for identification and authentication of bioactive compounds. The fingerprinting patterns (Fig. 2 and Fig. 2a) generated in the present study can be used as a reference in quality control of drugs containing "safed musli" as the ingredient. The profiles may also be useful for reducing adulteration in commercial trade. Unlike conventional root cultures which are initiated from excised root tips our studies offer an alternative, time saving and efficient system for the production of tuberous roots and microshoots without depending on ruinous harvesting of the conservation reliant aphrodisiac herb Chlorophytum borivilianum.

\section{Acknowledgements}

Sincere thanks are due to Sri Tushar Maharaj of Ramakrishna Mission Ashram Narendrapur (West Bengal) for providing plant materials as and when required. Thanks are also due to Prof. S. Ghosal of Natreon-Inc Kolkata (India) and Prof. S. Jha (Dept of Botany, University of Calcutta) for providing authentic markers for HPTLC.

\section{References}

Acharya D, Mitaine-Offer AC, Kaushik N, Miyamoto T, Paululat T and Dubois-Lacaille MA (2008) Furostane type steroidal saponins from the roots of Chlorophytum borivilianum. Helvetica Chimica Acta 91: 2262-2269.

Acharya D, Mitaine-Offer AC, Kaushik N, Miyamoto T, Paululat T, Mirojlet JF, Duchamp O and Dubois-Lacaille MA (2009) Cytotoxic spirostane type saponins from the roots of Chlorophytum borivilianum. J. Nat. Products 72: 177-181.

Basu S and Jha T (2007) In vitro propagation of the rare medicinal plant Chlorophytum borivilianum Sant. et. Fern. In: Proc. Nat. Symp of Plant. Biotechnology 285-289.

Boradhust CL and Duke JA (1998) Inside Plants: Arginine for impotence. The herb Companion. http:// www.herbcompanion.com

Bordia PC, Joshi A and Simlot MM (1995) Safed Musli. In: Chadha KL and Gupta R (eds) Advances in Horticulture (Vol. 11). Medicinal and Aromatic Plants. New Delhi. Malhotra Publishing house. pp. 429-451.

Bottger M (1978) Levels of endogenous indole-3-acetic acid and abscisic acid during the course of the formation of lateral roots. Zeitschrift für Pflanzenphysiologie. 86(4): 283-286.

Chi HJ, Shin KH, Kim HS and Cho HJ (1989) Production of ginseng saponin with cell culture. Korean J. Pharmacognosy 20: 162-169.

Choi KT, Ahn IO and Park JC (1994) Production of ginseng saponin in tissue culture of ginseng (Panax ginseg C.A. Mayer). Russian J. Plant Physiol. 41: 784-788.

Dave A, Bilochi G and Purohit SD (2003) Scaling up production and field performance of micropropagated medicinal herb 'Safed Musli' (Chlorophytum borivilianum). In vitro Cell. Dev. Biol. Pl. 39: 419-424. 
Furuya T, Yoshikawa T, Ishii T and Kajii K (1983) Regulation of saponin production in callus cultures of Panax ginseng. Planta Med. 47: 200-204.

Ghosh B, Mukherjee S, Jha TB and Jha S (2002) Enhanced colchicine production in root cultures of Gloriosa superba by direct and indirect precursors of the biosynthetic pathway. Biotechnol. Letters. 24: 231-234.

Ghosh S, Ghosh B and Jha S (2006) Aluminium chloride enhances colchicine production in root cultures of Gloriosa superba. Biotechnol. Letters 28: 497-503.

Ghosh S, Ghosh B and Jha S (2007) In vitro tuberisation of Gloriosa superba L. on basal medium. Sci. Hortic. 114(3): 220-223.

Goodwin PB and Morris SC (1979) Application of phytohormones to pea roots after removal of the apex: effect on lateral root production. Aust. J. Plant Physiol. 6: 195-200.

Hess B and Sherma J (2004) Quantification of Arginine in diatery supplement tablets and capsules by silica gel High Performance Thin Layer Chromatography with visible mode densitometry. Acta Chromatographica 14: 60-69.

Hill PE and Lawrence Jr. RH (1985) Hybrid Brassica seed production. US Patent No.: 4499687.

Hwang B, Ko KM, Hwang KH, Hwang SJ and Kong YH (1991) Production of saponins by hairy root cultures of ginseng (Panax ginseng CA Mayer) transformed with Agrobacterium rhizogenes. Korean J. Biotechnol. 34: 289-296.

Jin JM, Zhang YJ and Yang CR (2004) Cytotoxic steroidal saponins from Polygonatum zanlanscianense. J. Nat. Prod. 67(12): 1992-1995.

Kongbangkerd A and Wawrosch C (2003) Improved shoot regeneration from nodules of Charybdis numidica in a temporary immersion system. J. Hort. Sci. Biotech. 78(5): 650-655.

Maiti S and Geetha KA (2005) Characterization, genetic improvement and cultivation of Chlorophytum borivilianum - an important medicinal plant of India. Plant Genetic Resources 3(2): 264-272.

Maiti S and Geetha KA (2009) Safed Musli. Published by Indian Council of Agricultural Research (ICAR) New Delhi. pp. 18-39.

Mimaki Y, Kanmoto T, Shasida Y, Nishino A, Satomi Y and Nishino H (1996) Steroidal saponins from the underground parts of Chlorophytum comosum and their inhibitory activity on tumor promoter-induced phospholipids metabolism of Hela cells. Phytochemistry 41: 1405-1410.

Narasimhan S, Govindarajan R, Vijayakumar M, Thakur M, Dixit VK, Mehrotra S and Madhusudan KP (2006) Action of fructo-oligo polysaccharide fraction of Chlorophytum borivilianum against streptozotocin induced oxidative stress. Planta Med. 72: 1421-1424.

Norton ME and Boe AA (1982) In vitro propagation of ornamental Rosaceous plants. Hort. Sci. 17: 190-191.

Okrslar V, Plaper I, Kovac M, Erjavec A, Obermajer T, Rebec A, Ravnikar M and Zel J (2007) Saponins in tissue culture of Primula veris L. In vitro Cell. Dev. Biol. Pl. 43: 644-651. 
Oudhia P and Tripathi RS (1999) Scope of cultivation of important medicinal plants in Chattisgarh plains. In: Proc National Conference of healthcare and development of herbal medicines. pp. 71-78.

Purohit SD, Dave A and Kukda G (1994) Micropropagation of safed musli (Chlorophytum borivilianum), a rare Indian medicinal herb. Pl. Cell Tiss. Org. Cult. 39: 93-96.

Qiu S, Li XC, Xiong Y, Dong Y, Chai H, Farnsworth NR, Pezzuto JM and Fong HHS (2000) Isolation and characterization of cytotoxic saponin chloromaloside A from Chlorophytum malayense. Planta Med. 66: 587-590.

Rizvi MZ, Kukreja AK and Khanuja SPS (2007) In vitro culture of $C$. borivilianum Sant. et Fernanad. in liquid culture medium as a cost-effective measure. Curr. Sci. 92(1): 87-90.

Sahu NP, Banerjee S, Mondal NB and Mandal D (2008) Steroidal saponins. In: Progress in the chemistry of organic natural products. (Springer, Vienna). 89: 45-141.

Shilpa K, Selvakkumar C, Senthil AK and Lakshmi BS (2010) In vitro root culture of Ocimum sanctum $\mathrm{L}$. and evaluation of its free radical scavenging activity. Pl. Cell. Tiss. Org. Cult. 101: 105-109.

Sparg SG, Light ME and Staden J (2004) Biological activities and distribution of plant saponins. J. Ethnopharmacol. 94: 219-243.

Suri SS, Jain S, Arora DK and Ramawat KG (1999) In vitro high frequency regeneration of plantlets and tuberous root formation in Chlorophytum borivilianum. Gartenbauwissenschaft 64: 106-110.

Tandon M and Shukla YN (1997) Arundinoside A: a new spirostane saponin from C. arundinaceum. Journal of Indian Chemical Society 74: 56-58.

Thakur M, Bhargava S and Dixit VK (2007) Immunomodulatory Activity of Chlorophytum borivilianum Sant. et Fern. Evidence-Based Complementary and Alternative Medicine. 4(4): 419-423.

Thakur M and Dixit VK (2008) A review on some important medicinal plants of Chlorophytum spp. Pharmacognosy Reviews 2(3): 168-172.

Wawrosch C, Kongbangkerd A, Kopf A and Kopp B (2005) Shoot regeneration from nodules of Charybdis species. A comparison of semi solid, liquid and temporary immersion systems. Pl. Cell. Tiss. Org. Cult. 81(3): 319-322.

Wightman F, Schneider EA and Thimann KV (1980) Hormonal factors controlling the initiation and development of lateral roots. II. Effects of exogenous factors on lateral root formation in pea roots. Physiol. Plant 49: 304-314.

Wu J and Zhong JJ (1999) Production of ginseng and its bioactive components in plant cell culture: Current technological and applied aspects. J. Biotechnol. 68: 89-99.

Xin Di, Chan KKC, Leung HW and Huie CW (2003) Fingerprint profiling of acid hydrolyzates of polysachharides extracted from the fruiting bodies and spores of Linghi by high performance thin layer chromatography. Journal of Chromatography A. 1018: 85-95.

Yoshikawa T and Furuya T (1987) Saponin production by cultures of Panax ginseng transformed with Agrobacterium rhizogenes. Plant Cell Rep. 6: 449-454. 